\title{
Ki-67 and p53 Expression in Minimal Deviation Melanomas as Compared with Other Nevomelanocytic Lesions
}

Joe A. Chorny, M.D., Ronald J. Barr, M.D., Ainura Kyshtoobayeva, M.D., James Jakowatz, M.D., Richard J. Reed, M.D.

Dermatopathology Laboratory (JAC, RJB) and Department of Surgery (JJ), University of California Irvine Medical Center, Orange, California; Oncotech (AK), Irvine, California; and Reed Pathology Laboratory (RJR), New Orleans, Louisiana

Minimal deviation melanoma is a controversial entity encompassing a heterogeneous group of lesions cytologically in the spectrum between recognized subtypes of nevi and conventional "primary configuration" melanomas and reported to have a better prognosis than the latter. To evaluate the distinctiveness of minimal deviation melanoma, Ki-67 proliferation rates and p53 expression in minimal deviation melanomas were compared with those in compound nevi, Spitz nevi, and vertical growth phase superficial spreading malignant melanoma. Twelve examples of each lesion were immunostained with antibodies to the Ki-67 and p53 proteins and evaluated by a pathologist who was blind to the diagnoses. The mean Ki-67 (MIB-1) proliferation rates for the compound nevi, Spitz nevi, minimal deviation melanomas, and superficial spreading malignant melanomas were $0,3 \%, 13 \%$, and $25 \%$, respectively. The mean Ki-67 proliferation rate was statistically greater in the minimal deviation melanomas than in the compound nevi or the Spitz nevi $(P<.05)$, but the proliferation rates in the two melanoma subtypes were not statistically significant $(P=.08)$. The mean p53 values for these lesions were $0,9 \%, 9 \%$, and $26 \%$, respectively; the latter two were statistically different $(P<.01)$. Based on these Ki-67 and p53 immunophenotypes, minimal deviation melanoma may represent a distinct entity.

Copyright () 2003 by The United States and Canadian Academy of Pathology, Inc.

VOL. 16, NO. 6, P. 525, 2003 Printed in the U.S.A.

Date of acceptance: March 26, 2003.

Address reprint requests to: Ronald J. Barr, M.D., Dermatopathology Laboratory, University of California Irvine Medical Center, Building 52, Route 98, 101 The City Drive, South, Orange, CA 92868-3201; fax: 714-4568859; e-mail: RJBarr@UCI.edu.

DOI: 10.1097/01.MP.0000072747.08404.38
KEY WORDS: Compound nevus, MIB-1 (Ki-67) antibody, Minimal deviation melanoma, p53 protein, Spitz nevus, Skin, Superficial spreading malignant melanoma.

Mod Pathol 2003;16(6):525-529

In a busy dermatopathology practice, there are seen rare and unusual forms of nevomelanocytic proliferations that do not conform to generally recognized nevus or melanoma classifications. Placement of these problematic lesions into benign or malignant categories is difficult, and terms such as melanocytic tumor of undetermined malignant potential (1) or atypical melanocytic proliferation have been developed to communicate this prognostic uncertainty. Along these lines, the classification of minimal deviation melanoma (2) was developed for a subset of such tumors, composed of a monotonous cell population, that were more atypical than ordinary nevi but less atypical than conventional melanomas. A minimal deviation melanoma architecturally consists of an expansive nodule in the vertical growth phase that fills the papillary dermis and often extends into the reticular dermis (Fig. 1). The tumors are further classified, based on the cytologic characteristics, into subtypes such as a Spitz variant, a halo nevus-like variant, a pigmented spindle cell variant, a desmoplastic variant, a small cell variant, and a dermal variant. Minimal deviation melanomas have been associated with a better prognosis (2). As with other attempts to deal with these rare and difficult melanocytic neoplasms, this classification remains controversial (3) and has not been uniformly accepted in the dermatopathology community.

This study seeks to evaluate the distinctiveness of minimal deviation melanoma by comparing Ki-67 proliferation rates and p53 expression in minimal deviation melanomas with those in compound 


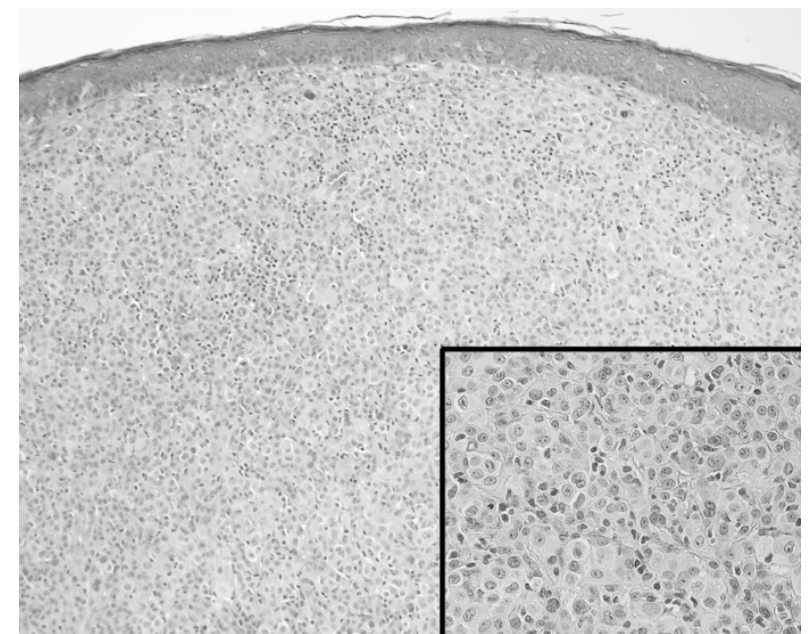

FIGURE 1. A minimal deviation melanoma with an expansive nodule filling the papillary dermis.

nevi, Spitz nevi, and vertical growth phase superficial spreading malignant melanomas.

The MIB-1 (anti-Ki-67) antibody has been used to identify cells in the proliferative cell cycle (G1, S, $\mathrm{G} 2$, and $\mathrm{M}$ phases) during which the Ki-67 protein is expressed. This protein is absent in the resting (G0) phase. Studies (4-10) of the Ki-67 proliferative rate in nevomelanocytic lesions have generally shown that compound nevi have low proliferative rates (usually $<3 \%$; range, $0-5 \%$ ), Spitz nevi have slightly higher and more variable proliferative rates (usually $<7 \%$; range, $0-50 \%$ ), and conventional melanomas have higher and more variable proliferative rates (in previous studies, means of $10-30 \%$ and ranges of 2-90\%).

The p53 protein is a $53-\mathrm{kDa}$ tumor suppressor nuclear phosphoprotein that acts as a negative regulator of cell proliferation (11). The wild-type p53 protein is rapidly degraded in normal cells and is usually present in very small quantities. Missense mutations or posttranslational modifications lead to an abnormally stable but inactive p53 protein that effectively promotes cell division and is detectable by immunohistochemistry. Studies (9-17) of p53 expression in nevomelanocytic lesions have shown similar results as with Ki-67. Compound nevi usually have little or no p53 expression, Spitz nevi occasionally express p53, and superficial spreading malignant melanoma express p53 in a highly variable percentage of cases $(0-97 \%$, reviewed in 17). Significant correlations between p53 immunoreactivity and the clinical course have been reported $(13,14)$.

\section{MATERIALS AND METHODS}

Twelve cases each of compound nevi, Spitz nevi, minimal deviation melanomas, and superficial spreading malignant melanomas were retrieved from the 1997-2000 files at the Dermatopathology Laboratory, University of California Irvine Medical Center. All 12 minimal deviation melanomas had been reviewed previously and diagnosed by one of the authors (RJR) who is a main proponent and pioneer of the concept of minimal deviation melanoma. The superficial spreading malignant melanomas were at least Clark's Level III and $\geq 1.1 \mathrm{~mm}$ thick. The tissue was fixed in $10 \%$ neutral buffered formaldehyde solution for 12 to 60 hours and processed routinely. Sections $4 \mu \mathrm{m}$ thick were cut for $\mathrm{H} \& \mathrm{E}$ and immunohistochemical staining, the latter performed by Oncotech (Irvine, CA). The immunoperoxidase procedure followed an avidin-biotin complex technique. Sections were stained using MIB-1 antibodies (1:200 dilution; Immunotech, Marseille, France) and anti-p53 antibodies (DO-1; 1:200 dilution; Santa Cruz Biotechnology, Santa Cruz, CA). Diaminobenzidine was the chromogenic substrate. Sections were counterstained with hematoxylin. Endogenous peroxidase was quenched by incubating the sections for 10 minutes with $0.3 \%$ hydrogen peroxide in methanol. Antigen retrieval with microwave heat for four successive 5-minute intervals was used with both antibodies. Sections of known tissues were used as positive controls. The omission of the primary antibodies and the replacement with normal sera served as negative controls.

The percentage of Ki-67- and p53immunostained cells was determined in each case by counting cells in contiguous high-power fields (400× magnification), using a cell counter until 200 cells were assessed. This was performed by a pathologist (JAC) who was blind to the diagnoses. Quantitative immunostain differences were assessed by a two-tailed Student's $t$ test using Microsoft Excel software. Clinical follow-up for evidence of metastatic spread was sought in the minimal deviation melanoma cases by contacting the primary dermatology office and the regional UCIMC melanoma clinic.

\section{RESULTS}

The mean Ki-67 proliferation rates for the compound nevi, Spitz nevi, minimal deviation melanomas, and superficial spreading malignant melanomas were $0,3 \%, 13 \%$, and $25 \%$, respectively (Table 1). The distribution of immunostained cells within each neoplasm varied among the subtypes of nevomelanocytic lesions (Fig. 2). The compound nevi had negligible numbers of Ki-67-positive cells, and the Spitz nevi had between 0 and $5 \%$ positive cells. In these benign lesions, staining was generally superficially located. The minimal deviation melanomas had $5-50 \%$ Ki-67-positive cells, and the 


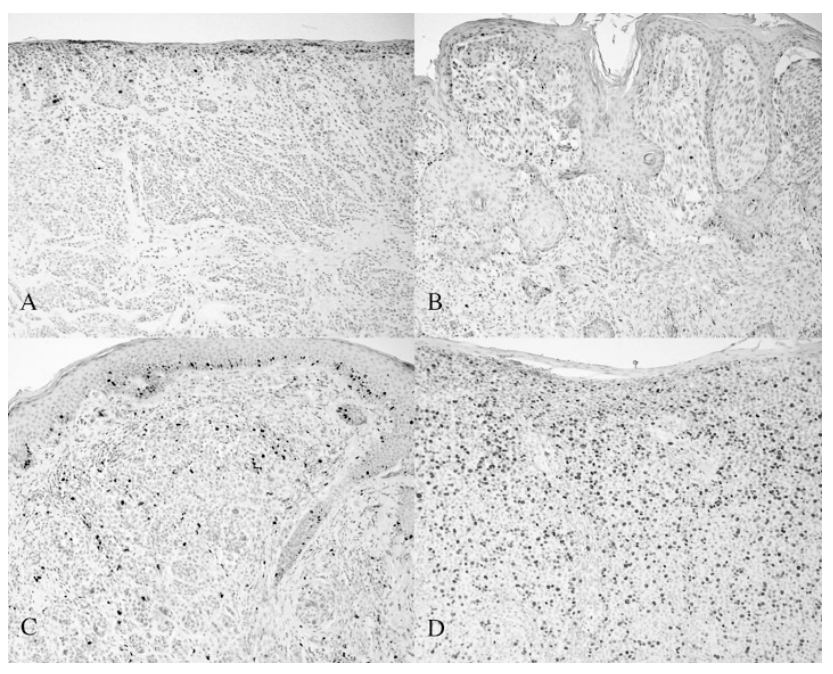

FIGURE 2. Immunohistochemistry for Ki-67 in (A) a compound nevus, (B) a Spitz nevus, (C) a minimal deviation melanoma, and (D) a vertical growth phase superficial spreading malignant melanoma.

mean minimal deviation melanoma Ki-67 proliferation rate was statistically greater than the mean proliferative rates of the compound and Spitz nevi $(P<.05)$. The superficial spreading malignant melanomas had from 5 to $62 \% \mathrm{Ki}-67-$ positive cells. In both melanoma subtypes, the positive cells were scattered at all levels throughout the tumor. The minimal deviation melanomas generally had lower proliferation rates than the superficial spreading malignant melanomas, but this difference was not statistically significant $(P=.08)$. There was considerable variability from case to case in the amount of Ki-67 and p53 immunostaining for both the minimal deviation melanomas and the superficial spreading malignant melanomas (Table 2).

The mean p53-positive fractions for the compound nevi, Spitz nevi, minimal deviation melanomas, and superficial spreading malignant melanomas were $0,9 \%, 9 \%$, and $25 \%$, respectively (Table $1)$. The distribution of immunostained cells was similar to that seen with Ki-67, with greater p53 staining superficially in the Spitz nevi and p53 immunostaining at all levels of the tumors in minimal deviation melanomas and superficial spreading malignant melanomas. The p53 immunostaining was statistically $(P<.01)$ less in the minimal devi-

TABLE 1. Percentage of Positive Ki-67 and p53 Cells

\begin{tabular}{|c|c|c|}
\hline \multirow{2}{*}{ Melanocytic Lesion } & $\begin{array}{c}\text { Ki-67 Proliferation } \\
\text { Rate }\end{array}$ & p53 Expression \\
\hline & Mean \pm SD [Range] & Mean \pm SD $[$ Range $]$ \\
\hline Compound nevi & $0.1 \pm 0.3$ & $0.3 \pm 0.9$ \\
\hline Spitz nevi & $2.8 \pm 1.2$ & $8.8 \pm 2.6$ \\
\hline Minimal Deviation Melanomas & $12.8 \pm 13.7 \quad[5-50]$ & $8.9 \pm 9.2$ \\
\hline $\begin{array}{l}\text { Superficial spreading } \\
\text { malignant melanomas }\end{array}$ & $25.3 \pm 18.8$ & $25.5 \pm 18.3 \quad[0-60]$ \\
\hline
\end{tabular}

ation melanomas than in the superficial spreading malignant melanomas.

Clinical follow-up for the minimal deviation melanoma was available in 7 of the 12 cases, and there were no reports of residual disease (Table 2).

\section{DISCUSSION}

This study contrasted immunostaining for the MIB-1 (Ki-67) proliferation marker and the p53 protein in minimal deviation melanomas with that in compound nevi, Spitz nevi, and superficial spreading malignant melanomas. With regard to Ki-67 proliferation rates (Table 1), those in minimal deviation melanomas (mean of $13 \%$ ) tended to be lower than did those in superficial spreading malignant melanomas (mean of 25\%); however, this difference was not statistically significant $(P=.08)$. This may have been due to the small sample size, but there was also considerable variation in the proliferation fractions among both subsets of melanomas, resulting in overlapping immunophenotypes. Both the minimal deviation melanomas and superficial spreading malignant melanomas had higher rates of proliferation than the compound nevi or Spitz nevi. Ki-67 protein expression in the compound nevi was negligible, and there was minor $(\leq 5 \%)$ expression in the Spitz nevi. The immunostained cells in these lesions tended to be superficially located, whereas the immunostained cells in both melanoma subtypes were evenly distributed at all levels throughout the tumors.

The Ki-67 proliferation rates and distribution patterns for compound nevi, Spitz nevi, and superficial spreading malignant melanomas described in this study are similar to those reported by previous investigators (4-10). For example, in the study by Kaleem et al. (10), the mean MIB-1 labeling percentages for compound nevi, Spitz nevi, and vertical growth phase melanomas were $0.5 \%, 1.4 \%$, and $16.1 \%$, respectively. McNutt et al. (8) studied nevoid melanoma and reported similar results to those of this study, with benign nevi, Spitz nevi, and nevoid melanoma having average MIB-1 proliferation rates of $<1 \%, 1-4 \%$, and $13 \%$, respectively. They also noted the predominance of MIB-1 staining in the superficial portions of benign nevi, as contrasted with immunostaining at all levels in the nevoid melanomas. Reactivity with proliferation markers can be fixation- and immunostain-technique dependent, leading to different degrees of staining across multiple laboratories; intralaboratory studies need to be performed for meaningful results.

In this study, expression of the p53 protein in the minimal deviation melanomas (mean of 9\%) was significantly less than that seen in the superficial spreading malignant melanomas (mean of $26 \%$; $P$ 
TABLE 2. Minimal Deviation Melanoma (MDM) Cases with Subtype, Percentage of Positive Ki-67 and p53 Cells, and Clinical Follow-Up

\begin{tabular}{|c|c|c|c|c|}
\hline MDM Case \# & MDM Subtype & Ki-67\% & p53\% & $\begin{array}{l}\text { Patient Follow-Up } \\
\text { (years) }\end{array}$ \\
\hline 1 & Dermal MDM & 50 & 20 & $\mathrm{~N} / \mathrm{A}$ \\
\hline 2 & Pigmented spindle cell MDM & 10 & 0 & $\mathrm{~N} / \mathrm{A}$ \\
\hline 3 & Desmoplastic MDM & 5 & 0 & $\mathrm{~N} / \mathrm{A}$ \\
\hline 4 & Halo MDM & 11 & 0 & No residual MDM (5) \\
\hline 5 & Spitz MDM & 7 & 10 & No residual MDM (5) \\
\hline 6 & Pigmented spindle cell MDM & 6 & 2 & N/A \\
\hline 7 & Dermal MDM & 8 & 10 & No residual MDM (5) \\
\hline 8 & Spitz MDM & 6 & N/A & N/A \\
\hline 9 & Spitz MDM & 8 & 4 & No residual MDM (4) \\
\hline 10 & Spitz MDM & 5 & 24 & No residual MDM (3) \\
\hline 11 & Spitz MDM & 31 & 19 & No residual MDM (3) \\
\hline 12 & Dermal type MDM & 7 & N/A & No residual MDM (3) \\
\hline
\end{tabular}

$<.01)$. There was negligible expression in the compound nevi, but there was moderate expression in the Spitz nevi (mean of 9\%) at the level seen in the minimal deviation melanomas.

The p53 immunostain results for compound nevi, Spitz nevi, and superficial spreading malignant melanomas in this study can be compared with those from previous studies (9-17) with mostly similar results. For example, Kaleem et al. (10) reported mean p53 labeling percentages for compound nevi, Spitz nevi, and vertical growth phase melanomas of 0,0 , and $12.6 \%$, respectively. Other studies have shown negligible p53 expression in compound nevi (14). We noted p53 expression in 5 of the 12 (42\%) Spitz nevi, and others have also reported p53 expression in a minority of Spitz nevi $(14,15)$. McGregor et al. (14) found p53 immunoreactivity in one of seven (14\%) Spitz nevi, and Bergman et al. (15) detected p53 in 2 of 26 (8\%) of Spitz nevi. In our Spitz nevi, p53 expression was variable and, in a few cases, quite elevated and did not parallel the Ki-67 proliferation rates. The reason for this is unclear, but this finding emphasizes caution in evaluating this antigen as a marker of malignant progression. There was p53 expression in 7 of 10 minimal deviation melanomas and in 11 of 12 superficial spreading malignant melanomas, and in both melanoma subsets the percentage of positive cells was often low $(<20 \%)$, which has been reported (17).

Clinical follow-up of our dozen minimal deviation melanomas confirmed that this proposed category of nevomelanocytic tumors has a generally favorable prognosis, with no reports of metastases in the seven cases for which clinical follow-up was available (Table 2). There are cases of minimal deviation melanoma in the files at UCIMC that have metastasized, including one in an immunocompromised patient, but these cases were not selected in our random study sample.

Unlike epithelial malignancies, melanomas are difficult to grade on the basis of the H\&E-stained section alone. The minimal deviation melanoma category was developed as a "working" classifica- tion for malignant melanomas with unusual features that suggested a low risk of metastases. To support this concept, one would expect less Ki-67 and p53 immunostaining in minimal deviation melanomas as compared with in conventional melanomas. In this study, the Ki-67 proliferation rates tended to be lower in minimal deviation melanomas than in superficial spreading malignant melanomas, although this difference was not statistically significant $(P=.08)$. The melanomas in both subtypes showed considerable variation in proliferation rates, which contributed to the lack of statistical significance. With regard to p53, its expression was lower in the minimal deviation melanomas as compared with in the superficial spreading malignant melanomas $(P<.01)$. This may have relevance to the theory that minimal deviation melanoma are partially transformed neoplasms that have not completed the mutational multistep progression to conventional nodular melanoma (8).

From these observations, minimal deviation melanoma may represent a form of low-grade melanoma. However, these results do not exclude the possibility that the minimal deviation melanoma category simply represents a mixture of benign nevus variants and conventional melanomas. Further study of minimal deviation melanomas will be necessary to clarify these issues. In particular, comparative genomic hybridization studies may determine whether minimal deviation melanomas have proportionately fewer genomic mutations as compared with the case of conventional melanomas (18).

In summary, the observations in this study arrived at by using the Ki- 67 and p53 immunostains offer adjunctive support for the subclassification of minimal deviation melanoma and, with due caution, may allow discrimination of individual cases from benign nevomelanocytic tumors and conventional melanomas.

Acknowledgments: The authors thank John Valone, M.D. for his assistance in retrieving case material. 


\section{REFERENCES}

1. Elder D, Elenitsas R. Benign pigmented lesions and malignant melanoma. In: Elder D, Elenitsas R, Jaworsky C, Johnson B Jr, editors. Lever's histopathology of the skin. 8th ed. Philadelphia: Lippincott-Raven; 1997. p. 625-84.

2. Reed RJ. Minimal deviation melanoma: borderline and intermediate neoplasia. Clin Lab Med 2000;20:745-58.

3. LeBoit PE. Minimal deviation melanoma: concept or quagmire? Adv Dermatol 1997;13:289-303.

4. Hofmann-Wellenhof R, Rieger E, Smolle J, Kerl H. Proliferative activity in Spitz naevi compared with other melanocytic skin lesions. Melanoma Res 1993;3:313-7.

5. Rudolph P, Schubert C, Schubert B, Parwaresch R. Proliferation marker Ki-S5 as a diagnostic tool in melanocytic lesions. J Am Acad Dermatol 1997;37:169-78.

6. Ling-Xi LL, Crotty KA, McCarthy SW, Palmer AA, Krik JJ. A zonal comparison of MIB1-Ki67 immunoreactivity in benign and malignant melanocytic lesions. Am J Dermatopathol 2000;22:489-95.

7. Smolle J, Soyer HP, Kerl H. Proliferative activity of cutaneous melanocytic tumors defined by Ki- 67 monoclonal antibody. A quantitative immunohistochemical study. Am J Dermatopathol 1989;11:301-7.

8. McNutt SN, Urmacher C, Hakimian J, Hoss DM, Lugo J. Nevoid malignant melanoma: morphologic patterns and immunohistochemical reactivity. J Cutan Pathol 1995;22:50217.

9. Kanter-Lewensohn L, Hedblad MA, Wejde J, Larsson O. Immunohistochemical markers for distinguishing Spitz nevi from malignant melanomas. Mod Pathol 1997;10:917-20.

10. Kaleem Z, Lind AC, Humphrey PA, Sueper RH, Swanson PE, Ritter JH, et al. Concurrent Ki-67 and p53 immunolabeling in cutaneous melanocytic neoplasms: an adjunct for recognition of the vertical growth phase in malignant melanomas? Mod Pathol 2000;13:217-22.

11. Campbell C, Quinn AB, Ro Y-S, Angus B, Rees JL. p53 mutations are common and early events that precede tumor invasion in squamous cell neoplasia of the skin. J Invest Dermatol 1993;100:746-8.

12. Cristofolini M, Boi S, Girlando S, Zumiani G, Cristofolini P, Palma PD, et al. p53 protein expression in nevi and melanomas. Arch Dermatol 1993;129:739-43.

13. Korabiowska M, Brinck U, Hoenig JF, Bartkowski SB, Kellner S, Marx D, et al. Significance of p53 antigen in malignant melanomas and naevi of the head and neck area. Anticancer Res 1995;15:885-9.

14. McGregor JM, Yu CC, Dublin EA, Barnes DM, Levison DA, MacDonald DM. p53 immunoreactivity in human malignant melanoma and dysplastic naevi. Br J Dermatol 1993;128: 606-11.

15. Bergman R, Shemer A, Levy R, Friedman-Birnbaum R, Trau $\mathrm{H}$, Lichtig C. Immunohistochemical study of p53 protein expression in Spitz nevus as compared with other melanocytic lesions. Am J Dermatopathol 1995;17:547-50.

16. Sparrow LE, Soong R, Dawkins HJ, Iacopetta BJ, Heenan PJ. p53 gene mutation and expression in naevi and melanomas. Melanoma Res 1995;5:93-100.

17. Saenz-Santamaría M, McNutt S, Bogdany JK, Shea CR. p53 expression is rare in cutaneous melanomas. Am J Dermatopathol 1995;17:344-9.

18. Bastian BC, LeBoit PE, Hamm H, Broecker E-B, Pinkel D. Chromosomal gains and losses in primary cutaneous melanomas detected by comparative genomic hybridization. Cancer Res 1998;58:2170-5. 